\title{
Multi-Sector Beamforming with MMSE Receiver for Spatially Correlated Channel
}

\author{
Jae-Heung Yeom and Yong-Hwan Lee \\ School of Electrical Engineering and INMC \\ Seoul National University \\ Seoul, Korea \\ e-mail: jhyeom@ttl.snu.ac.kr, ylee@snu.ac.kr
}

\begin{abstract}
The use of statistical eigen-beamforming is effective in spatially correlated fading environments, but it may suffer from interference near the sector boundary when applied to the downlink with universal frequency reuse. This interference effect may not sufficiently be handled by a minimum mean square error (MMSE) receiver unless a sufficient number of receive antennas are employed. In this paper, we consider the use of multi-sector beamforming that cooperates with a neighboring sector in the same cell to mitigate this interference problem. By exploiting long-term channel state information (CSI), the proposed scheme can obtain transmit array gain without the use of instantaneous CSI, while avoiding interference from the adjacent cooperating sector. The performance of the proposed scheme is analyzed with combined use of an MMSE receiver and verified by computer simulation.
\end{abstract}

Keywords-beamforming; interference; multi-sector; correlation

\section{INTRODUCTION}

Demand for higher throughput has motivated advanced wireless systems such as the 3GPP LTE and mobile-WiMAX to employ multi-cell configuration with universal frequency reuse [1], [2]. Various measurement results show that multiantenna channels are often spatially correlated in real environments [3]. The use of eigen-beamforming is quite effective with low feedback signaling overhead in spatially correlated channel [4], [5]. However, it may cause users near the cell boundary to experience serious inter-cell interference in universal frequency reuse environments [6]. In particular, when the cell is divided into a number of sectors, users near the sector boundary may experience weak received signal strength (RSS) from the serving sector due to sector antenna pattern as well as interference from adjacent sectors [1], [7]. Advanced wireless systems consider the use of multiple receive antennas in the mobile station (MS) [2], [8], enabling the use of a minimum mean square error (MMSE)-type receiver to suppress interference while reducing the fading effect [9]. However, when the number of strong interferers is larger than the number of receive antennas minus one, the output signal-tointerference-plus-noise power ratio (SINR) of the MMSE receiver may significantly deteriorate [10].

To improve the performance of users near the sector/cell boundary, the use of base station (BS) coordination has recently been considered, where the BSs share the channel state information (CSI) to minimize the interference effect [11], [12].
However, this BS cooperation may incur signaling overhead increasing in exponetially proportional to the number of coordinating BSs and suffer from performance degradation due to the signaling delay. Recently, null beamforming technique has been introduced, where the interference to other co-channel cells is removed by exchanging instantaneous inter-cell CSI [13]. However, it requires a considerable amount of instantaneous CSI and cannot cancel out the interference due to the use of a single receive antenna. It is quite practical and easy to cooperate among sectors in the same cell, because information between these sectors can be exchanged without signaling overhead [8].

The performance near the sector boundary can also be improved without the exchange of inter-cell information with the aid of softer handover, macro diversity handover or fast sector selection (FSS) with muting [8], [14]. In the macrodiversity handover, users can demodulate the signal without interfering with each other, while obtaining frequency diversity gain. The FSS with muting allows only one sector in better condition to transmit data signal with doubled transmit power, while making the other sector, called muted sector, sleep to avoid mutual interference. However, these schemes do not consider the use of multiple transmit antennas and spatial correlation.

In this paper, we design a multi-sector beamforming scheme that cooperates with an adjacent sector in the same cell. The proposed scheme can avoid interference from the adjacent sector in the same cell, while enhancing the transmit array gain using long-term statistics of the channel. It does not require additional signaling overhead compared to conventional singlesector beamforming schemes. When applied to the use of an MMSE receiver, the performance is analyzed in the presence of arbitrary-power interferers.

Following Introduction, Section II describes the system model in consideration. Section III presents the proposed multisector beamforming and analyzes its performance. The performance of the proposed scheme is verified by computer simulation in Section IV. Finally, conclusions are given in Section V.

\section{SYSTEM MODEL}

Consider the downlink of a cellular system that comprises $N$ hexagonal cells each of which comprises $S$ sectors (i.e., a 
total of $S \cdot N$ sectors). For ease of description, it is assumed that each sector uses $M$ transmit antennas and the MS uses two receive antennas. Assuming that the target user is under service from sector $m$, the received signal of the target user can be represented as

$$
\begin{aligned}
\mathbf{y} & =\alpha_{m} \mathbf{H}_{m} \mathbf{w}_{m} x_{m}+\sum_{i \neq m} \alpha_{i} \mathbf{H}_{i} \mathbf{w}_{i} x_{i}+\mathbf{n} \\
& =\alpha_{m} \mathbf{H}_{m} \mathbf{w}_{m} x_{m}+\sum_{i \in \Omega, \neq m} \alpha_{i} \mathbf{H}_{i} \mathbf{w}_{i} x_{i}+\mathbf{z}
\end{aligned}
$$

where $x_{i}$ denotes the data of sector $i$ with unit average power, $\alpha_{i}$ denotes the received signal strength (RSS) from sector $i$, $\mathbf{H}_{i}$ is the $(2 \times M)$ channel matrix from sector $i, \mathbf{w}_{i}$ denotes an $(M \times 1)$ beam weight from sector $i, \mathbf{n}$ is the $(2 \times 1)$ additive white Gaussian noise (AWGN) vector, and $\Omega$ denotes an active set comprising sectors defined by

$$
\Omega=\left\{\hat{k} \mid \frac{\alpha_{k}^{2}}{\alpha_{m}^{2}} \geq \delta, \quad 0 \leq k \leq S \cdot N-1\right\} .
$$

Here $\delta$ denotes a threshold value for the active set, and $\mathbf{z}$ denotes the $(2 \times 1)$ interference plus noise vector except the interference from sectors belonging to $\Omega$, which can be assumed to be zero-mean complex Gaussian with covariance $E\left\{\mathbf{z z}^{*}\right\}=N_{z} \mathbf{I}_{2}$ [15], where $\mathbf{I}_{2}$ denotes a $(2 \times 2)$ identity matrix. The beam weight $\mathbf{w}_{i}$ is determined by the eigenvector corresponding to the maximum eigenvalue of the channel covariance matrix. It is assumed that $\left\|\mathbf{w}_{i}\right\|^{2} \leq 1$, where $\|\cdot\|$ denotes the Frobenius norm [16].

\section{Proposed Multi-Sector BeAmforming}

Consider the use of multi-sector beamforming with cooperation between sectors in the same cell to enhance the performance near the sector boundary. The multi-sector beamforming configuration can be extended from $(M \times 2)$ to $(2 M \times 2)$ antenna configuration by concatenating two adjacent sectors.

Assume that the BS transmits the target user signal through sector $m$ and $m^{\prime}$. Then, the received signal can be represented as

$$
\mathbf{y}=\alpha_{m} \mathbf{H}_{D} \mathbf{w}_{D} X_{m}+\sum_{i \in \Omega, \neq m, m^{\prime}} \alpha_{i} \mathbf{H}_{i} \mathbf{w}_{i} X_{n}+\mathbf{z}
$$

where $\mathbf{w}_{D}=\left[\begin{array}{ll}\mathbf{w}_{m}{ }^{T} & \mathbf{w}_{m^{\prime}}{ }^{T}\end{array}\right]^{T}$ and $\mathbf{H}_{D}$ are respectively the $(2 M \times 1)$ beamforming vector and $(2 \times 2 M)$ channel matrix from sector $m$ and $m^{\prime}$ to the target user, given by

$$
\mathbf{H}_{D}=\left[\begin{array}{ll}
\mathbf{H}_{m} & \mathbf{H}_{m^{\prime}}
\end{array}\right] \boldsymbol{\Lambda}_{D}
$$

Here $\boldsymbol{\Lambda}_{D}$ denotes the normalized RSS matrix represented in an $(2 M \times 2 M)$ diagonal matrix whose first and last $M$ diagonal elements are all one and $\alpha_{m^{\prime}} / \alpha_{m}$, respectively, and the superscript $T$ denotes transpose. Note that the transmit power of $\mathbf{w}_{D}$ is limited by

$$
\left\|\mathbf{w}_{D}\right\|^{2} \leq \max \left(\operatorname{tr}\left[\boldsymbol{\Lambda}_{D}^{2}\right] / M\right)=\max \left(1+\alpha_{m^{\prime}}^{2} / \alpha_{m}^{2}\right)=2
$$

where $\operatorname{tr}[\cdot]$ denotes the trace of a matrix. It can be seen that $\left\|\mathbf{w}_{D}\right\|^{2}=2$ when the cooperating sectors have the same RSS (i.e., $\alpha_{m}=\alpha_{m^{\prime}}$ ).

The channel covariance matrix can be represented as

$$
\begin{aligned}
\mathbf{R} & \triangleq E\left\{\mathbf{H}_{D}^{*} \mathbf{H}_{D}\right\} / 2 \\
& =\boldsymbol{\Lambda}_{D}\left(E\left\{\left[\begin{array}{ll}
\mathbf{H}_{m} & \mathbf{H}_{m^{\prime}}
\end{array}\right]^{*}\left[\begin{array}{ll}
\mathbf{H}_{m} & \mathbf{H}_{m^{\prime}}
\end{array}\right]\right\} / 2\right) \boldsymbol{\Lambda}_{D}
\end{aligned}
$$

where $E\{\cdot\}$ denotes the expectation, the superscript $*$ denotes transpose conjugate. It can be seen that the RSS of two cooperating sectors changes the channel covariance. Since $\mathbf{R}$ is Hermitian and positive definite, it can be decomposed as [18]

$$
\mathbf{R}=\mathbf{Q} \Sigma^{2} \mathbf{Q}^{*}
$$

where $\mathbf{Q}=\left[\begin{array}{lll}\mathbf{q}_{1} & \cdots & \mathbf{q}_{2 M}\end{array}\right]$ is an $(2 M \times 2 M)$ unitary matrix whose columns $\left\{\mathbf{q}_{k}\right\}$ are the eigenvectors of $\mathbf{R}$, and $\boldsymbol{\Sigma}^{2}$ is a $(2 M \times 2 M)$ diagonal matrix whose diagonal terms are descending-ordered eigenvalues (i.e., $\lambda_{1} \geq \cdots \geq \lambda_{2 M}$ ) of $\mathbf{R}$. The channel matrix can be represented as [18]

$$
\mathbf{H}_{D}=\mathbf{H}_{w} \mathbf{R}^{1 / 2}
$$

where $\mathbf{R}^{1 / 2}$ denotes the square root of $\mathbf{R}$ and $\mathbf{H}_{w}$ is the $(2 \times 2 M)$ spatially white complex Gaussian channel matrix [16].

The beam weight $\mathbf{w}_{D}$ can be given by

$$
\mathbf{w}_{D}=\sqrt{1+\alpha_{m^{\prime}}^{2} / \alpha_{m}^{2}} \mathbf{q}_{1}
$$

Thus, the effective channel of the desired signal can be represented by 


$$
\begin{aligned}
\mathbf{H}_{D} \mathbf{w}_{D} & =\sqrt{1+\alpha_{m^{\prime}}^{2} / \alpha_{m}^{2}} \mathbf{H}_{w} \mathbf{R}^{1 / 2} \mathbf{q}_{1} \\
& =\sqrt{1+\alpha_{m^{\prime}}^{2} / \alpha_{m}^{2}} \sqrt{\lambda_{1}} \mathbf{h}_{w} .
\end{aligned}
$$

where $\mathbf{h}_{w}$ is the $(2 \times 1)$ spatially white complex Gaussian channel vector [16]. The output SINR of an MMSE receiver can be represented by [9]

$$
\gamma=\alpha_{m}^{2}\left(\mathbf{H}_{D} \mathbf{w}_{D}\right)^{*} \mathbf{K}^{-1}\left(\mathbf{H}_{D} \mathbf{w}_{D}\right)
$$

where $\mathbf{K}$ denotes the covariance matrix of the interference plus noise, defined as

$$
\mathbf{K}=\sum_{i \in \Omega, \neq m, m^{\prime}} \alpha_{i}^{2}\left(\mathbf{H}_{i} \mathbf{w}_{i}\right)\left(\mathbf{H}_{i} \mathbf{w}_{i}\right)^{*}+N_{z} \mathbf{I} .
$$

Assuming that the major interference is from only sector $m^{\prime}$, the multi-sector beamforming can avoid this major interference (i.e., $\mathbf{K}=N_{z} \mathbf{I}_{2}$ ). In this case, the average output SINR can be given by

$$
\begin{aligned}
E\{\gamma\} & =\frac{\alpha_{m}^{2}}{N_{z}} E\left\{\left(\mathbf{H}_{D} \mathbf{w}_{D}\right)^{*}\left(\mathbf{H}_{D} \mathbf{w}_{D}\right)\right\} \\
& =2 \lambda_{1} \frac{\left(\alpha_{m}^{2}+\alpha_{m^{\prime}}^{2}\right)}{N_{z}}
\end{aligned}
$$

However, when the interference comes from other cells, it is required to consider the covariance $\mathbf{K}$ of the interference plus noise. In this case, the probability density function (pdf) of the output SINR $\gamma$ can be represented as [19]

$$
p(\gamma)=\sum_{k=1}^{2} B_{k} \exp \left(-E\left\{\sigma_{k}\right\} \gamma\right)
$$

where

$$
B_{k}=\prod_{i=1}^{2} E\left\{\sigma_{i}\right\} /\left(\prod_{i=1, \neq k}^{2}\left(E\left\{\sigma_{i}\right\}-E\left\{\sigma_{k}\right\}\right)\right)
$$

and $\sigma_{k}$ denotes the eigenvalue of $\mathbf{K G}^{-1}$. Here, $\mathbf{G}$ denotes the covariance of the target signal, given by

$$
\begin{aligned}
\mathbf{G} & =\alpha_{m}^{2} E\left\{\left(\mathbf{H}_{D} \mathbf{w}_{D}\right)\left(\mathbf{H}_{D} \mathbf{w}_{D}\right)^{*}\right\} \\
& =\lambda_{1}\left(\alpha_{m}^{2}+\alpha_{m^{\prime}}^{2}\right) E\left\{\mathbf{h}_{w} \mathbf{h}_{w}^{*}\right\}=\lambda_{1}\left(\alpha_{m}^{2}+\alpha_{m^{\prime}}^{2}\right) \mathbf{I}_{2} .
\end{aligned}
$$

It can be seen from (14) that the pdf of the output SINR can be determined by the mean eigenvalue of $\mathbf{K G}^{-1}$. Since $\mathbf{w}_{i}$ in (12) is an $M$-dimensional unit-norm vector, the effective channel from sector $i \in \Omega, \neq m, m^{\prime}$ can be represented as

$$
\mathbf{H}_{i} \mathbf{w}_{i}=\varepsilon_{i} \mathbf{h}_{w, i}
$$

where $\mathbf{h}_{w, i}$ is a $(2 \times 1)$ spatially white Gaussian channel vector defined by [16]

$$
E\left\{\mathbf{h}_{w, k}^{*} \mathbf{h}_{w, i}\right\}= \begin{cases}0, & k \neq i \\ 2, & k=i\end{cases}
$$

$$
\varepsilon_{i}=\sqrt{E\left\{\left(\mathbf{H}_{i} \mathbf{w}_{i}\right)^{*}\left(\mathbf{H}_{i} \mathbf{w}_{i}\right)\right\} / 2}=\sqrt{\mathbf{w}_{i}^{*} E\left\{\mathbf{H}_{i}^{*} \mathbf{H}_{i} / 2\right\} \mathbf{w}_{i}} .
$$

Since $\varepsilon_{i}^{2}$ corresponds to the Rayleigh quotient of the covariance matrix of $\mathbf{H}_{i}$, it belongs to a range between the minimum and the maximum eigenvalue [17]. Thus, $\mathbf{K}$ can be rewritten as

$$
\mathbf{K}=\mathbf{C}_{I} \mathbf{P}_{I} \mathbf{C}_{I}^{*}+N_{z} \mathbf{I}_{2} .
$$

In the presence of $L$ interferences from sectors $\left\{j_{k} \in \Omega ; \neq m, m^{\prime}, k=1, \cdots, L\right\}, \mathbf{C}_{I}$ is a $(2 \times L)$ random matrix comprising $L$ interference vectors as columns, i.e.,

$$
\mathbf{C}_{I}=\left[\begin{array}{llll}
\mathbf{h}_{w, j_{1}} & \mathbf{h}_{w, j_{2}} & \cdots & \mathbf{h}_{w, j_{L}}
\end{array}\right]
$$

and $\mathbf{P}_{I}$ is an $(L \times L)$ diagonal matrix whose $k$-th diagonal elements are $\alpha_{j_{k}}^{2} \cdot \varepsilon_{j_{k}}^{2}$. Letting $E\left\{\beta_{k}\right\}$ be the mean eigenvalue of $\mathbf{C}_{I} \mathbf{C}_{I}^{*}$, it can be shown that [20]

$$
\begin{gathered}
E\left\{\beta_{1}\right\}=\frac{L\left(L^{2}+3\right)}{2^{L}}+\sum_{k=2}^{L-1} \frac{1}{2^{2 L-k-1}}\left(\begin{array}{c}
2 L-k-1 \\
L-2
\end{array}\right)\left(\begin{array}{l}
k \\
2
\end{array}\right) \\
E\left\{\beta_{2}\right\}=2 L-E\left\{\beta_{1}\right\} .
\end{gathered}
$$

Letting $\left\{\mu_{k}\right\}$ be the eigenvalue of $\mathbf{C}_{I} \mathbf{P}_{I} \mathbf{C}_{I}^{*}$ in (20) when the interferers have arbitrary power, it can be shown that

$$
\begin{aligned}
\sum_{k=1}^{2} E\left\{\mu_{k}\right\} & =E\left\{\sum_{k=1}^{2} \mu_{k}\right\}=E\left\{\operatorname{tr}\left[\mathbf{C}_{I} \mathbf{P}_{I} \mathbf{C}_{I}^{*}\right]\right\} \\
& =\sum_{k=1}^{L} \alpha_{j_{k}}^{2} \varepsilon_{j_{k}}^{2} E\left\{\left\|\mathbf{c}_{j_{i}}\right\|^{2}\right\}=2 \sum_{k=1}^{L} \alpha_{j_{k}}^{2} \varepsilon_{j_{k}}^{2} .
\end{aligned}
$$

Since [17] 


$$
\begin{aligned}
\prod_{k=1}^{2} \mu_{k} & =\operatorname{det}\left[\mathbf{C}_{I} \mathbf{P}_{I} \mathbf{C}_{I}^{*}\right] \\
& =\operatorname{det}\left[\mathbf{C}_{I}\right]^{2} \operatorname{det}\left[\mathbf{P}_{I}\right]=\prod_{k=1}^{2} \beta_{k} \operatorname{det}\left[\mathbf{P}_{I}\right]
\end{aligned}
$$

it can be approximated as

$$
\prod_{k=1}^{2} E\left\{\mu_{k}\right\} \approx \prod_{k=1}^{2} E\left\{\beta_{k}\right\} \operatorname{det}\left[\mathbf{P}_{I}\right]
$$

where $\operatorname{det}[\cdot]$ denotes the determinant of a matrix. Similarly, it can be shown that

$$
\begin{aligned}
\sum_{k=1}^{2} E\left\{\sigma_{k}\right\} & =E\left\{\operatorname{tr}\left[\mathbf{K G}^{-1}\right]\right\} \\
& =\operatorname{tr}\left[\left\{E\left\{\mathbf{C}_{I} \mathbf{P}_{I} \mathbf{C}_{I}^{*}\right\}+N_{z} \mathbf{I}_{2}\right\} \mathbf{G}^{-1}\right] \\
& =\left\{\sum_{k=1}^{L} \alpha_{j_{k}}^{2} \varepsilon_{j_{k}}^{2}+N_{z}\right\} \operatorname{tr}\left[\mathbf{G}^{-1}\right] .
\end{aligned}
$$

Since [17]

$$
\begin{aligned}
\prod_{k=1}^{2} \sigma_{k} & =\operatorname{det}\left[\mathbf{K G}^{-1}\right] \\
& =\operatorname{det}\left[\mathbf{C}_{I} \mathbf{P}_{I} \mathbf{C}_{I}^{*}+N_{z} \mathbf{I}_{2}\right] \operatorname{det}\left[\mathbf{G}^{-1}\right] \\
& =\prod_{k=1}^{2}\left(\mu_{k}+N_{z}\right) \operatorname{det}\left[\mathbf{G}^{-1}\right]
\end{aligned}
$$

it can be approximated as

$$
\prod_{k=1}^{2} E\left\{\sigma_{k}\right\} \approx \prod_{k=1}^{2}\left(E\left\{\mu_{k}\right\}+N_{z}\right) \operatorname{det}\left[\mathbf{G}^{-1}\right]
$$

Thus, the average output SINR can be approximated as

$$
\begin{aligned}
E\{\gamma\}= & \int_{0}^{\infty} \gamma p(\gamma) d \gamma \approx \frac{\sum_{k=1}^{2} E\left\{\sigma_{k}\right\}}{\prod_{k=1}^{2} E\left\{\sigma_{k}\right\}} \\
= & \frac{\sum_{i \in \Omega, \neq m, m^{\prime}} \alpha_{i}^{2} \varepsilon_{i}^{2}+N_{z}}{\prod_{k=1}^{2}\left(E\left\{\mu_{k}\right\}+N_{z}\right)} \cdot \frac{\operatorname{tr}\left[\mathbf{G}^{-1}\right]}{\operatorname{det}\left[\mathbf{G}^{-1}\right]} .
\end{aligned}
$$

It can further be approximated using (24) and (26) as

$$
\frac{\sum_{i \in \Omega, \neq m, m^{\prime}} \Gamma_{i}+1}{\left(E\left\{\beta_{1}\right\} E\left\{\beta_{2}\right\} / N_{z}^{2}\right) \prod_{i \in \Omega, \neq m, m^{\prime}} \alpha_{i}^{2} \varepsilon_{i}^{2}+2 \sum_{i \in \Omega, \neq m, m^{\prime}} \Gamma_{i}+1} \cdot \gamma_{\text {target }}
$$

where $\Gamma_{i}\left(=\alpha_{i}^{2} \varepsilon_{i}^{2} / N_{z}\right)$ denotes the average SNR of sector $i$ and $\gamma_{\text {target }}\left(=\operatorname{tr}\left[\mathbf{G}^{-1}\right] /\left(N_{z} \operatorname{det}\left[\mathbf{G}^{-1}\right]\right)\right)$ is the average SNR of the target signal. The first term in the denominator of (31) represents the degradation due to the interference.

Assuming that users near the cell/sector boundary experience interference at most from two sources, the multisector beamforming can avoid interference from the adjacent sector, making users experience a single dominant interference (i.e., $\Gamma_{j_{1}} \gg 1$ ). The average output SINR of the MMSE receiver increases in proportion to the average SNR, i.e.,

$$
\begin{aligned}
E\{\gamma\} & \approx \frac{\Gamma_{j_{1}}+1}{\left(E\left\{\beta_{1}\right\} E\left\{\beta_{2}\right\} / N_{z}\right) \Gamma_{j_{1}}+2 \Gamma_{j_{1}}+1} \cdot \gamma_{\text {target }} \\
& \approx \frac{\gamma_{\text {target }}}{2}
\end{aligned}
$$

where $E\left\{\beta_{1}\right\}=2$ and $E\left\{\beta_{2}\right\}=0$. It can be shown that

$$
\gamma_{\text {target }}=\frac{\operatorname{tr}\left[\mathbf{G}^{-1}\right]}{N_{z} \operatorname{det}\left[\mathbf{G}^{-1}\right]}=\frac{2 \lambda_{1}\left(\alpha_{m}^{2}+\alpha_{m^{\prime}}^{2}\right)}{N_{z}} .
$$

Thus, the use of multi-sector beamforming can achieve large transmit array gain and power gain as well as interference cancellation compared to the use of a single sector beamforming.

\section{Performance Evaluation}

The analytic design and performance of the proposed beamforming scheme are verified by computer simulation. The proposed scheme is applied to a $(2 \times 2)$ MIMO configuration in correlated MIMO fading channel with covariance matrix $\Delta(\rho)$ given by

$$
[\Delta(\rho)]_{p, q}=\rho^{|p-q|} \exp \left(j(p-q) \frac{\pi}{12}\right)
$$

where $\rho$ denotes the magnitude of the correlation coefficient between two adjacent transmitter antennas, and $[\cdot]_{p, q}$ denotes the element of the $p$-th row and the $q$-th column. That is, $E\left\{\left[\begin{array}{ll}\mathbf{H}_{m} & \mathbf{H}_{m^{\prime}}\end{array}\right]^{*}\left[\begin{array}{ll}\mathbf{H}_{m} & \mathbf{H}_{m^{\prime}}\end{array}\right]\right\} / 2=\boldsymbol{\Delta}(\rho) \cdot \varepsilon_{i}^{2}$ can be set to one assuming that $\mathbf{w}_{i}$ is independent of $\mathbf{H}_{i}$. It is also assumed that sector 0 allocates the resource to the target user at each frame. 
The performance is evaluated in terms of the geometry defined by

$$
\begin{aligned}
G & =\frac{\alpha_{0}^{2}}{\sum_{i \in \Omega, \neq 0} \alpha_{i}^{2}+\sum_{i \in \Omega} \alpha_{i}^{2}+N_{0}}=\frac{\alpha_{0}^{2}}{\sum_{i \in \Omega, \neq 0} \alpha_{i}^{2}+N_{z}} \\
& =\frac{\Gamma_{0}}{\sum_{i \in \Omega, \neq 0} \Gamma_{i}+1} .
\end{aligned}
$$

In the following figures, the legend 'BF_MMSE', 'BF_MRC', 'MSBF_MMSE', 'SHO_MMSE', 'MD_MMSE', and 'FSS_MMSE' denote the statistical eigen-beamforming with MMSE receiver, the statistical eigen-beamforming with maximum ratio combining (MRC), the multi-sector beamforming with MMSE receiver, the softer handover with MMSE receiver, the macro diversity handover with MMSE receiver, and the FSS with muting and MMSE receiver, respectively. Also 'EBF_MMSE' denotes the eigenbeamforming with MMSE receiver, which transmits the signal with the strongest eigenmode of the instantaneous CSI [2], causing a considerable amount of feedback signaling burden.

Figure 1 depicts the analytic and simulation results according to $\Gamma_{0}$, assuming a single interference from adjacent sector 1 with the same RSS as the serving sector (i.e., $\Omega=\{0,1\}$ and $\left.\Gamma_{1}=\Gamma_{0}\right)$. In this case, the multi-sector beamforming can avoid the interference from the adjacent sector while increasing the transmit array gain and doubling the transmit power gain. It can be seen that the MMSE receiver increases the average output SINR in proportion to $\Gamma_{0}$, while the MRC can not mainly due to dominant interference. It can also be seen that the analytic results agree well with the simulation results. The larger $\rho$, the higher the performance of the proposed scheme due to the increase of the transmit array gain. It can be seen that the statistical eigen-beamforming provides performance similar to the eigen-beamforming with instantaneous CSI. Thus, the proposed scheme with the longterm CSI is effective in spatially correlated channel with a marginal increase of feedback signaling burden. Figure 2 depicts the analytic and simulation results according to $\Gamma_{0}$ in the presence of two interferences; one from adjacent sector 1 and the other from sector 2 (i.e., $\Omega=\{0,1,2\}$, and $\Gamma_{1}=\Gamma_{0}$ and $\Gamma_{2}=\Gamma_{0}+2 \mathrm{~dB}$ ). It can be seen that the MMSE receiver with the single-sector beamforming schemes ('BF_MMSE' and 'EBF_MMSE') suffer from untreated interference, while the MMSE receiver with the multi-sector beamforming works well by properly removing the dominant interference. It can be seen that the average output SINR of the multi-sector beamforming increases in proportion to $\Gamma_{0}$ and that the gap between the single-sector and the multi-sector beamforming increases as the SNR increases. Figure 3 and 4 depict the performance in 19cell environments (with $S=3$ ), where the cell radius is $1 \mathrm{~km}$, the path loss follows $28.6+35 * \log 10(d), d$ is the distance (in meters) between the sector and the user, and the sector antenna pattern follows $70^{\circ}$ (-3dB beamwidth) with a front-to-back ratio of $20 \mathrm{~dB}$ [7]. It is assumed that sectors having an RSS
TABLE I. THE SNR OF SERVING AND NEIGHBORING SECTORS NEAR THE SECTOR BOUNDARY.

\begin{tabular}{|l|l|l|}
\hline \multicolumn{1}{|c|}{$G(\mathbf{d B})$} & \multicolumn{1}{|c|}{$\Omega$} & \multicolumn{1}{c|}{ SNR (dB) } \\
\hline \hline-0.5 & $\{0,1\}$ & $\Gamma_{1}=\Gamma_{0}=9.1$ \\
\hline-1 & $\{0,1\}$ & $\Gamma_{1}=\Gamma_{0}=5.7$ \\
\hline-2 & $\{0,1\}$ & $\Gamma_{1}=\Gamma_{0}=2.1$ \\
\hline-4 & $\{0,1,11\}$ & $\Gamma_{1}=\Gamma_{0}=1.3, \Gamma_{11}=0.3$ \\
\hline-5 & $\{0,1,11\}$ & $\Gamma_{1}=\Gamma_{0}=0.1, \Gamma_{11}=1.0$ \\
\hline-7 & $\{0,1,11\}$ & $\Gamma_{1}=\Gamma_{0}=-1.8, \Gamma_{11}=2.2$ \\
\hline
\end{tabular}

TABLE II. THE SNR OF THE SERVING AND NEIGHBORING SECTORS AT A GEOMETRY OF $-5 \mathrm{~dB}$.

\begin{tabular}{|l|l|l|}
\hline \multicolumn{1}{|c|}{ Direction $\left(^{\circ}\right)$} & \multicolumn{1}{|c|}{$\Omega$} & \multicolumn{1}{c|}{ SNR (dB) } \\
\hline \hline 60 & $\{0,1,11\}$ & $\Gamma_{0}=\Gamma_{1}=0.1, \Gamma_{11}=1.0$ \\
\hline 58 & $\{0,1,11\}$ & $\Gamma_{0}=-0.1, \Gamma_{1}=-1.4, \Gamma_{11}=1.5$ \\
\hline 56 & $\{0,1,11\}$ & $\Gamma_{0}=-0.1, \Gamma_{1}=-2.8, \Gamma_{11}=2.0$ \\
\hline 54 & $\{0,11\}$ & $\Gamma_{0}=-1.6, \Gamma_{1}=-4.3, \Gamma_{11}=0.8$ \\
\hline
\end{tabular}

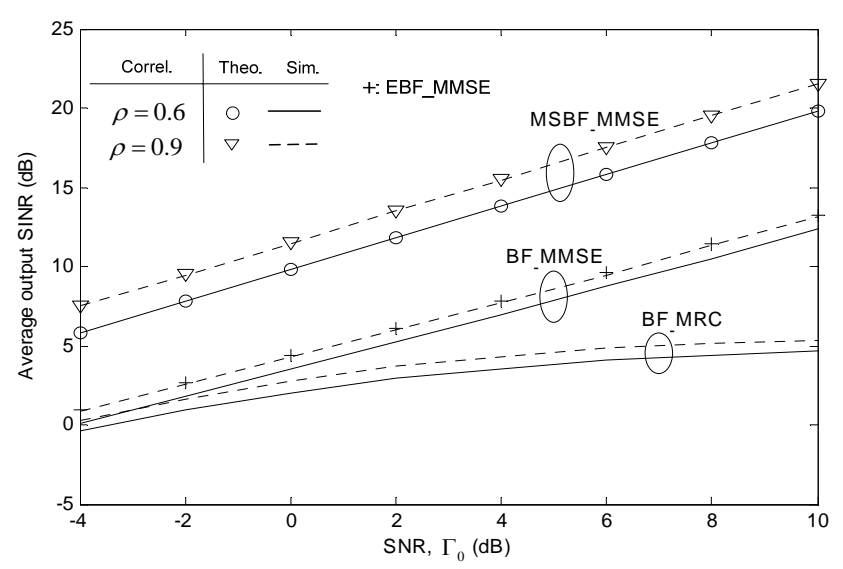

Figure 1. Performance in the presence of interference from a single adjacent sector.

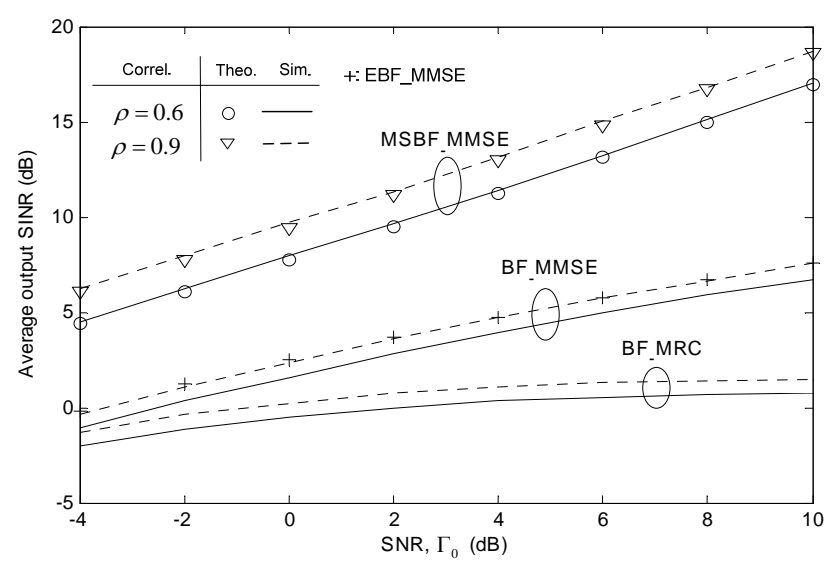

Figure 2. Performance in the presence of interference from two adjacent sectors. 


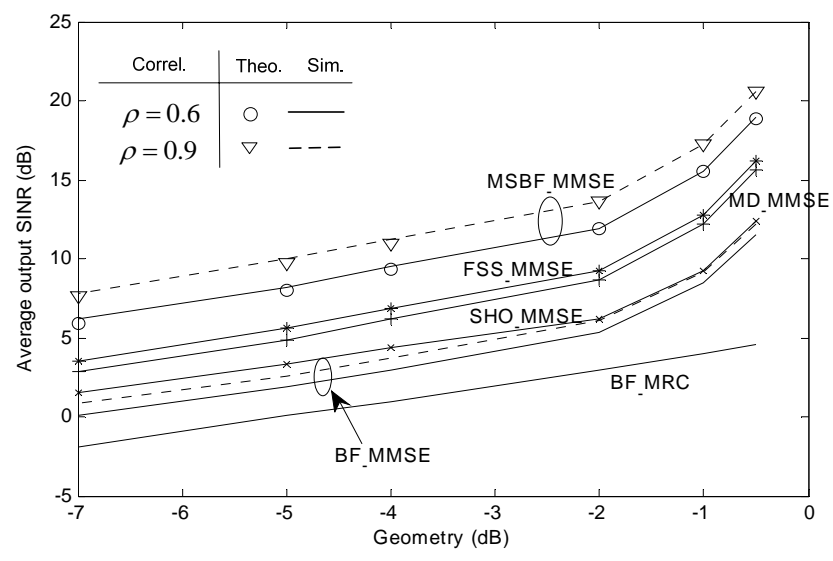

Figure 3. Performance of users near the sector boundary.

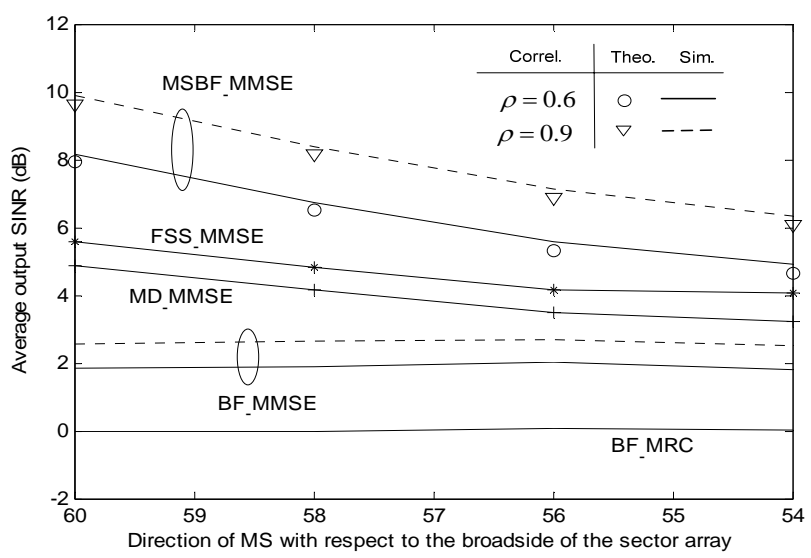

Figure 4. Performance according to the direction of user at a geometry of $-5 \mathrm{~dB}$.

larger than one half that of the serving sector belong to the active set (i.e., $\delta=-3 \mathrm{~dB}$ ), and that the target user is located near the boundary between sector 0 and 1 and experiences interference form sector 11 at the same time. Table I summarizes the active set and the corresponding SNR. It can be seen from Figure 3 that the multi-sector beamforming outperforms the FSS with muting and the macro diversity handover mainly due to the transmit array gain through beamforming. Figure 4 depicts the performance when users are located near the cell boundary at a direction of between $54^{\circ}$ and $60^{\circ}$ with respect to the broadside of the sector array, and experience geometry of $-5 \mathrm{~dB}$ at all directions. It can be seen from Table II that the RSS of the adjacent sector (i.e., sector 1) decreases as the user moves away from the sector boundary. In this case, the multi-sector beamforming can not sufficiently obtain the transmit array gain and transmit power gain. Since the analytic results agree well with the simulation results, the operating point of the multi-sector beamforming can analytically be determined.

\section{CONCLUSIONS}

We have considered the use of multi-sector beamforming for the service of users near the sector boundary in the downlink of cellular systems in spatially correlated channel environments. The performance of the proposed multi-sector beamforming scheme combined with MMSE receiver has been analyzed in terms of the average output SINR. The simulation results show that the multi-sector beamforming is very effective for users near the sector boundary, outperforming conventional schemes such as the eigen-beamforming, softer handover, macro diversity handover and FSS with muting.

\section{REFERENCES}

[1] Y. Xiang, J. Luo and C. Hartmann, "Inter-cell interference mitigation through flexible resource reuse in OFDMA based communication networks," in Proc. European Wireless Conf., Apr. 2007.

[2] WiMAX Forum, "A comparative analysis of Mobile WiMAX deployment alternatives in the access network," May 2007.

[3] J. Kermoal, L. Schumacher, K. Pedersen, P. Mogensen and F Frederiksen, "A stochastic MIMO radio channel model with experimental validation," IEEE J. Select. Areas. Commun., vol. 20, no. 6, pp. 1211-1226, Aug. 2002.

[4] H. Sampath, V. Erceg and A. Paulraj, "Performance analysis of linear precoding based on field trials results of MIMO-OFDM system," IEEE Trans. Wirel. Commun., vol. 4, no. 5, pp. 404-409, Mar. 2005.

[5] J. Choi, S. Kim and I. Choi, "Statistics eigen- beamforming with selection diversity for spatially correlated OFDM downlink," IEEE Trans. Veh. Technol., vol. 56, pp. 2931-2940, Sept. 2007.

[6] WiMAX Forum, "Mobile WiMAX-Part I: A. Technical overview and performance evaluation,” Feb. 2006.

[7] 3GPP TR 25.996, "3GPP technical specification group radio access network; Spatial channel model for MIMO simulations,” V6.1.0, Sept. 2003.

[8] 3GPP TR 25.814, "3GPP technical specification group radio access network; Physical layer aspects for evolved UTRA,” V7.1.0, Sept. 2006.

[9] J. Winters, "Optimum combining in digital mobile radio with cochannel interference,” IEEE J. Select. Areas Commun., vol. 33, pp. 523-539, Aug. 1984.

[10] D. Tse and P. Viswanath, Fundamentals of Wireless Communication, Cambridge University Press, 2005.

[11] S. Shamai and B. Zaidel, "Enhancing the cellular downlink capacity via co-processing at the transmitting end,” in Proc. IEEE Veh. Technol. Conf., vol. 3, pp. 1745-1749, May 2001.

[12] S. Jafar, G. Foschini and A. Goldsmith, "PhantomNet: exploring optimal multicellular multiple antenna systems," in Proc. IEEE Veh. Technol. Conf., vol. 1, pp. 261-265, Sept. 2002.

[13] L. Shao and S. Roy, "Downlink multicell MIMO-OFDM: an architecture for next generation wireless networks," in Proc. IEEE WCNC., vol. 2, pp. 1120-1125, Mar. 2005.

[14] A. Morimoto, K. Higuchi and M. Sawahashi, "Performance comparison between fast sector selection and simultaneous transmission with softcombining for intra-node B macro diversity in downlink OFDM radio access,” in Proc. IEEE Veh. Technol. Conf., vol. 1, pp. 157-161, 2006.

[15] S. Plass, A. Dammann and S. Kaiser, "Analysis of coded OFDMA in a downlink multi-cell scenario,” in Proc. OFDM workshop (InOWo 2004), Sept. 2004.

[16] A. Paulraj, R. Nabar and D. Gore, Introduction to Space-Time Wireless Communications, Cambridge Universe Press, 2003.

[17] G. Strang, Linear Algebra and Its Applications, Harcourt Brace Jovanovich College Publishers, 1988.

[18] P. Amers, et al., "Survey of channel and radio propagation models for wireless MIMO systems,” EURASIP Jour. On Wireless Commun., ID 19070, 2007.

[19] T. Pham and K. Balmain, "Multipath performance of adaptive antennas with multiple interferers and correlated fadings," IEEE Trans. Veh. Technol., vol. 48, pp. 342-352, Mar. 1999.

[20] M. Chiani, M. Z. Win, A. Zanella, R. Mallik and J. Winters, "Bounds and approximations for optimum combining of signals in the presence of multiple cochannel interferers and thermal noise," IEEE Trans. Commun., vol. 51, no. 2, pp 296-307, Feb. 2003. 